\author{
М. А. Киселев ${ }^{1}$, Е. В. Земляная ${ }^{1}$, А. Ю. Грузинов ${ }^{2}$, \\ Е. И. Жабицкая ${ }^{1,4}$, О. М. Ипатова ${ }^{3}$, В. Л. Аксенов ${ }^{1,5}$
}

\title{
АНАЛИЗ ВЕЗИКУЛЯРНОЙ СТРУКТУРЫ НАНОЧАСТИЦ ФОСФОЛИПИДНОЙ СИСТЕМЫ ТРАНСПОРТА ЛЕКАРСТВ НА ОСНОВЕ ДАННЫХ МУРР
}

Направлено в журнал «Кристаллография»

${ }^{1}$ Объединенный институт ядерных исследований, Дубна

${ }^{2}$ European Molecular Biology Laboratory, DESY, Гамбург, Германия

${ }^{3}$ Научно-исследовательский институт биомедицинской химии им. В. Н. Ореховича, Москва

${ }^{4}$ Государственный университет «Дубна», Дубна, Россия

${ }^{5}$ Петербургский институт ядерной физики им. Б. П. Константинова Национального исследовательского центра «Курчатовский институт», Гатчина, Россия 
Киселев М. А. и др.

Анализ везикулярной структуры наночастиц фосфолипидной

системы транспорта лекарств на основе данных МУРР

Методом малоуглового рентгеновского синхротронного рассеяния проанализирована везикулярная структура фосфолипидной транспортной наносистемы (ФТНС) для трех значений концентрации ФТНС в воде: $25,31,25$ и $37,5 \%$. Рассчитаны параметры везикул: радиус, полидисперсность радиуса, толщина липидного бислоя, внутренняя структура липидного бислоя для двух моделей распределения плотности длины рассеяния фотона. Расчеты проводились двумя различными методами: по формфактору сферической неоднородной оболочки и по методу разделенных формфакторов. Два метода расчета спектров и два используемых приближения для описания внутренней структуры липидного бислоя дают совпадающие результаты, которые показывают уменьшение радиуса везикулы, толщины липидного бислоя и толщины области углеводородных цепочек при увеличении концентрации мальтозы в воде.

Работа выполнена в Лаборатории нейтронной физики им. И. М. Франка и Лаборатории информационных технологий ОИЯИ.

Препринт Объединенного института ядерных исследований. Дубна, 2017

Kiselev M. A. et al.

P3-2017-32

Analysis of Vesicular Structure of Nanoparticles

in the Phospholipid Based Drug Delivery System using SAXS Data

Small-angle X-ray scattering has been used to characterize the Phospholipid Transport Nanosystem (PTNS) at the PTNS concentrations in water of 25\%, 31.25\%, and $37.5 \%$. The vesicle's average radius, size polydispersity, bilayer thickness, and internal structure were evaluated from the experiment via applications of two models for the photon scattering density distribution. Two independent methods were used for the calculation of the SAXS spectra: the form factor of the heterogeneous spherical shell and the method of separated form factors. Two methods of the spectra calculation and two models for the approximation of the photon scattering length density evaluation provide coincident results, which demonstrate the decrease in the vesicle radius, thickness of the bilayer, and thickness of the hydrocarbon chains region at the increase in the maltose concentration in water.

The investigation has been performed at the Frank Laboratory of Neutron Physics and the Laboratory of Information Technologies, JINR. 


\section{ВВЕДЕНИЕ}

Один из способов повышения эффективности существующих лекарственных веществ - разработка для них систем транспорта с применением современных нанотехнологий. В настоящее время ряд готовых лекарственных форм на основе различных систем транспорта уже внедрен в медицинскую практику. Эти препараты отличаются высокой эффективностью и широко востребованы на рынке лекарственных средств.

Наибольший интерес представляют системы транспорта лекарственных веществ на основе фосфолипидов, так как они биодеградируемы, биологически инертны, не вызывают аллергических, антигенных или пирогенных реакций [1]. В отличие от мицелл везикулы на основе фосфолипидов позволяют транспортировать как водонерастворимые, так и водорастворимые молекулы лекарственных веществ, что делает их наиболее перспективным переносчиком [2].

Везикулы представляют собой полые частицы, содержимое которых ограничено одним или несколькими бислоями фосфолипидов. Диаметр везикул варьируется от $200 \AA$ А (однослойные везикулы, у которых стенка состоит из одного бислоя) до 10-50 мкм (многослойные везикулы, у которых стенка состоит из десятков, сотен или тысяч бислоев, - липосомы). Для разработки систем транспорта лекарственных веществ используют как однослойные, так и многослойные везикулы (липосомы). В последнее время для транспорта лекарственных соединений в основном используются частицы малых размеров, так как установлено, что уменьшение размера липосом в 8 раз продлевает их циркуляцию в кровотоке почти в 40 раз. Уменьшение размера частиц приводит к уменьшению площади их поверхности. Лекарственные вещества на основе таких наночастиц должны изменять свою фармакокинетику [3].

Лекарственная субстанция в зависимости от своего химического строения может либо встраиваться в гидрофобную область фосфолипидного бислоя, либо адсорбироваться на поверхности и/или включаться во внутреннюю область везикулы. Поэтому достоверная информация о размерах и структуре бислоя везикул является необходимой для их эффективного практического применения в качестве переносчика лекарственных веществ.

В Институте биомедицинской химии (ИБМХ) на основе растительных (соевых) фосфолипидов разработана технология получения транспортных наночастиц предельно малого размера (150-250 А̊) в виде лиофильно высушенного порошка, стабильного при хранении [4]. Показано, что встраивание 
некоторых лекарственных субстанций в эти наночастицы существенно увеличивает их биодоступность и терапевтическую эффективность [5]. В работе [6] путем сравнительного анализа спектров малоуглового синхротронного рентгеновского рассеяния (МУРР) на образцах вышеупомянутой фосфолипидной транспортной наносистемы (ФТНС) и «классической» везикулярной системы димиристоилфосфатидилхолина показано, что наночастицы ФТНС имеют морфологию однослойных везикул. В этой работе, а также в [7,8] на основе различных подходов сделаны предварительные оценки основных структурных параметров везикулярной системы ФТНС (средний радиус везикул, полидисперсность среднего радиуса, толщина бислоя).

В данной работе представлены результаты анализа структуры полидисперсной популяции однослойных везикул ФТНС по данным МУРР в зависимости от концентрации мальтозы в водном растворе.

\section{ЭКСПЕРИМЕНТ}

Метод малоуглового рассеяния нейтронов (МУРН) известен как весьма эффективный экспериментальный метод исследования структуры везикулярных систем [9-12]. При использовании МУРН применение тяжелой воды в качестве растворителя дает усиление контраста. Недостатком применения МУРН является некогерентный фон от ядер водорода в измеряемом образце [13]. Лиофилизированная ФТНС состоит из соевых фосфолипидов и мальтозы с весовым соотношением компонентов $1: 4$. Увеличение концентрации ФТНС в тяжелой воде ведет к увеличению концентрации недейтерированной мальтозы и, соответственно, увеличению некогерентного фона, что ограничивает возможность измерения малоуглового рассеяния в области больших значений вектора рассеяния. Тем самым ухудшается пространственное разрешение эксперимента при определении структуры липидного бислоя везикулы. МУРН наиболее эффективно применять при низких концентрациях ФТНС в воде - $20 \%$ по весу и менее.

Трудности при использовании МУРР для случая однослойных везикул в водной среде связаны с тем, что средняя плотность электронов в фосфолипидном бислое близка к плотности электронов в воде, что делает везикулу практически «невидимой» для фотонов. Добавление дисахаридов в водные растворы существенно увеличивает контраст (разность в плотности электронов в липидном бислое и водной среде) [14-17]. Например, интенсивность рассеяния от везикул димиристоилфосфатидилхолина в 40\%-м водном растворе сахарозы в 100 раз превышает интенсивность рассеяния от тех же самых везикул в воде [14]. Применение МУРР наиболее эффективно при концентрации ФТНС в воде, равной и выше $25 \%$. Начиная с концентрации мальтозы в воде $20 \%$ обеспечивается хороший контраст. При этом не происходит увеличения некогерентного фона. Малая величина некогерентного фона является существенным преимуществом рентгеновского синхротронного экспери- 
мента. Это позволяет получать более достоверную информацию о структуре липидного бислоя по сравнению с нейтронным экспериментом $[7,13]$.

ФТНС разработана в ИБМХ на основе растительных фосфолипидов и состоит из соевого фосфатидилхолина (95-97\%-й чистоты) и мальтозы в весовом соотношении $1: 4$. При растворении лиофилизата в воде образуется эмульсия фосфолипидных наночастиц в водном растворе мальтозы. Концентрация мальтозы в образце зависит от степени растворения, что позволяет варьировать рентгеновский контраст. Таким образом, методики контрастирования везикулярных систем дисахаридами, развитые в работах [14-17], открывают возможность исследования водных растворов ФТНС методом МУРР при их терапевтических концентрациях.

Образец приготавливали путем растворения лиофилизированного порошка ФТНС в воде с концентрацией препарата $25,31,25$ и $37,5 \%$. При этом концентрация мальтозы после растворения препарата составляла соответственно 20, 25 и 30\%. Наличие в образце 20-30\% дисахарида (мальтозы) обеспечивает необходимый контраст для проведения эксперимента методом МУРР.

Исследования ФТНС проводили на установке ДИКСИ Курчатовского источника синхронного излучения (КИСИ) в Национальном исследовательском центре «Курчатовский институт». Измерения проводили при расстояниях «образец-детектор» $L_{\mathrm{sd}}=30,56$ и 241,5 см. Длина волны фотона $\lambda=1,625$ А. Использовался двухмерный детектор MarCCD. В эксперименте измерялась зависимость интенсивности рассеянного излучения $I(q)$ от величины модуля вектора рассеяния $q=4 \pi \sin \left(\frac{\theta}{2}\right) / \lambda$, где $\theta-$ угол рассеяния фотона на образце.

\section{МОДЕЛЬ}

Интенсивность малоуглового рассеяния для монодисперсной популяции сферических везикул радиусом $R$ с бислоем толщиной $d$ записывается, как в [10]:

$$
I_{m}(q)=I_{\mathrm{o}} n A^{2}(q) S(q),
$$

где $I_{\mathrm{O}}$ - интенсивность фотонов в падающем на образец пучке; $n-$ количество везикул, см$^{3} ; A-$ амплитуда рассеяния фотона на одной везикуле, определяемая с учетом сферической симметрии везикулы следующим образом:

$$
A(q)=4 \pi \int_{0}^{\infty} \rho_{c}(r) \frac{\sin (q r)}{q r} r^{2} d r .
$$

Здесь $\rho_{c}=\rho-\rho_{0}-$ контраст между плотностью длины рассеяния липидным бислоем $\rho$ и плотностью длины рассеяния раствором $\rho_{0}$. Структурный 
фактор $S$ вычисляется по формуле Дебая [18]:

$$
S(q, R)=1-\frac{8 V_{\nu}}{\nu}\left(\frac{\sin (2 q R)}{2 q R}\right),
$$

где $V_{\nu}$ - объем везикулы; $\nu=1 / n ; R-$ радиус везикулы.

Факторизация исходного выражения для амплитуды рассеяния приводит к методу разделенных формфакторов (РФФ), существенно упрощающему вычисления, позволяя выбрать фактически любую подходящую функцию для моделирования плотности длины рассеяния. Метод РФФ успешно использовался для анализа различных везикулярных систем [13,19-22]. Подробное описание этого подхода дано, например, в [11-13]. Здесь мы ограничимся только основным выражением для интенсивности $I_{\mathrm{SFF}}$ :

$$
I_{\mathrm{SFF}}(q)=I_{0} n F_{s}(q, R) F_{b}(q, \rho) S(q, R),
$$

где $F_{s}$ и $F_{b}-$ формфакторы бесконечно тонкой сферической оболочки радиусом $R$ и липидного бислоя толщиной $d$. Формфакторы $F_{s}$ и $F_{b}$ имеют вид

$$
\begin{array}{r}
F_{s}(q, R)=\left(4 \pi \frac{R^{2}}{q R} \sin (q R)\right)^{2}, \\
F_{b}\left(q, \rho_{c}\right)=\left(\int_{-d / 2}^{d / 2} \rho_{c}(x) \cos (q x) d x\right)^{2} .
\end{array}
$$

Нужно отметить, что метод РФФ имеет ограничения, так как предназначен для анализа структуры везикул с большим радиусом и малой толщиной бислоя. Поскольку частицы ФТНС имеют малый размер, проведены вычисления на основе «полной» модели (ПМ), определяемой выражением (1), и на основе метода РФФ с использованием формул (4), (5). Как будет показано в следующем разделе, получены близкие результаты.

Среднеквадратичное отклонение $\sigma$ от среднего радиуса $\langle R\rangle$ везикул ФТНС рассчитывалось на основе распределения Шульца с коэффициентом $m$ :

$$
\begin{gathered}
G(R,\langle R\rangle)=\frac{R^{m}}{m !}\left(\frac{m+1}{\langle R\rangle}\right)^{m+1} \exp \left[-\frac{(m+1) R}{\langle R\rangle}\right], \\
\sigma=\sqrt{\frac{1}{(m+1)}} .
\end{gathered}
$$


Тогда макроскопическое сечение $I_{p}$ полидисперсной популяции везикул имеет вид

$$
I_{p}(q)=n \frac{\int_{R \min }^{R \max } I(q, R,\langle R\rangle) G(R,\langle R\rangle) d R}{\int_{R \min }^{R \max } G(R,\langle R\rangle) d R},
$$

где $I$ - интенсивность монодисперсной популяции, вычисляемая по формуле (1) или (4). Пределы интегрирования $R_{\min }$ и $R_{\max }$ полагались равными 10 и $800 \AA$ А соответственно.

Окончательное выражение для интенсивности макроскопического сечения $I$ формируется с учетом некогерентного фона $I_{B}$ и разрешения спектрометра [23]:

$$
I(q)=I_{p}(q)+\frac{1}{2} \Delta^{2} \frac{d^{2}}{d q^{2}} I_{p}(q)+I_{B} .
$$

Здесь $\Delta=6 \cdot 10^{-4} \AA^{-1}-$ второй момент функции разрешения спектрометра.

В расчетах использовались две представленные на рис. 1 модели плотности длины рассеяния $\rho$, учитывающие различное рассеяние фотонов в области полярных голов $(\mathrm{PH})$ и углеводородных цепочек $(\mathrm{CH})$. В одноступенчатой модели (рис. $1, a$ ) область углеводородных цепочек предполагается однородной. В двухступенчатой модели (рис. $1, \sigma)$ выделены области, соответствующие углеводородным цепочкам двух типов: $\mathrm{CH}_{2}$ и $\mathrm{CH}_{3}$. Значения $\rho_{\mathrm{CH}_{2}}$ и $\rho_{\mathrm{CH}_{3}}$ фиксированы и составляют $\rho_{\mathrm{CH}_{2}}=7,95 \cdot 10^{10} \mathrm{~cm}^{-2}, \rho_{\mathrm{CH}_{3}}=4,71 \cdot 10^{10} \mathrm{~cm}^{-2}$. Значения $\rho_{0}$ в зависимости от концентрации ФТНС в растворе даны в табл. 1 .

Свободными параметрами модели, подгоняемыми к экспериментальным данным, являются средний радиус $\langle R\rangle$, коэффициент полидисперсности $m$, толщина бислоя $d$, плотность длины рассеяния в области полярных голов $\rho_{\mathrm{PH}}$, толщина $D$ области углеводородных цепочек $\mathrm{CH}_{2}$, толщина $L$ области углеводородных цепочек $\mathrm{CH}_{3}$, плотность длины рассеяния в области поляр-

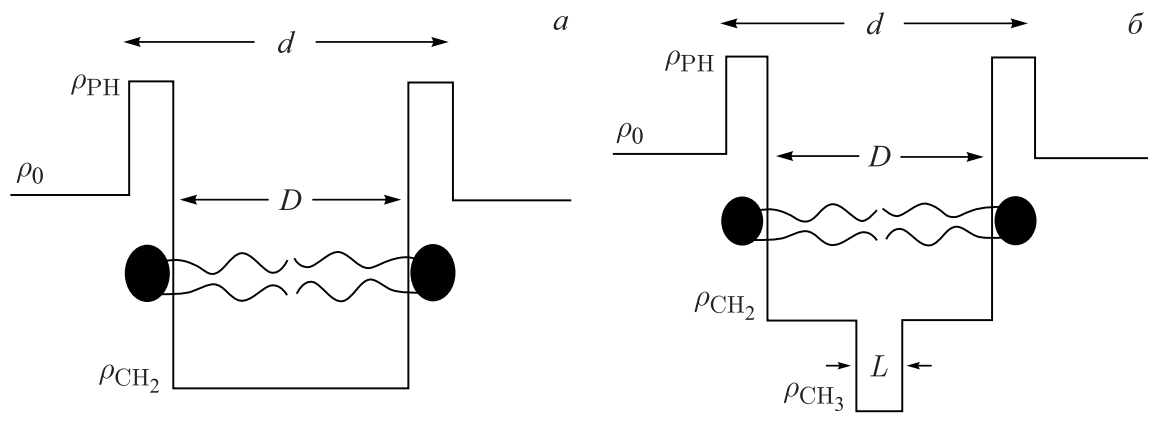

Рис. 1. Модели плотности длины рассеяния $\rho$ бислоем ФТНС 
Таблица 1. Значения концентрации мальтозы и соответствующие значения плотности длины рассеяния фотона в зависимости от концентрации ФТНС в воде при $t=20^{\circ} \mathbf{C}$

\begin{tabular}{|c|c|c|c|}
\hline $\begin{array}{c}\text { Концентрация } \\
\text { ФТНС }\end{array}$ & $\begin{array}{c}\text { Концентрация } \\
\text { мальтозы, \% }\end{array}$ & $\begin{array}{c}\text { Плотность, } \\
\text { г/см }^{3}\end{array}$ & $\begin{array}{c}\rho_{0}, \\
10^{10} \mathrm{~cm}^{-2}\end{array}$ \\
\hline 25 & 20 & 1,081 & 10,11 \\
\hline 31,25 & 25 & 1,100 & 10,27 \\
\hline 37,5 & 30 & 1,125 & 10,48 \\
\hline
\end{tabular}

ных голов $\rho_{\mathrm{PH}}$, некогерентный фон $I_{B}$, число везикул на единицу объема $n$. Эти параметры рассчитывались путем минимизации функции

$$
\chi^{2}=\frac{1}{N-k} \sum_{i=1}^{N}\left(\frac{I\left(q_{i}\right)-I_{\exp }\left(q_{i}\right)}{\delta\left(q_{i}\right)}\right)^{2},
$$

где $\delta\left(q_{i}\right)$ - погрешности экспериментальных данных; $N$ - число экспериментальных точек; $k$ - количество фитируемых параметров. Расчеты проводились на основе двух независимых подходов, обеспечивающих совпадающие результаты: с использованием программы DFUMIL из библиотеки JINRLIB (http://www.jinr.ru/programs/jinrlib), реализующей обобщенный метод наименьших квадратов [24], а также с использованием глобальной минимизации на основе метода асинхронной дифференциальной эволюции $[25,26]$.

\section{ОБСУЖДЕНИЕ РЕЗУЛЬТАТОВ}

Результаты расчетов параметров полидисперсной популяции однослойных везикул ФТНС в зависимости от концентрации мальтозы, полученные с использованием двух моделей бислоя, представлены в табл. 2 и 3 и на рис. 2 .

Таблица 2. Расчет на основе полной модели (ПМ) и модели РФФ параметров везикулярной системы ФТНС с одноступенчатой функцией распределения электронов (см. рис. 1, $a$ )

\begin{tabular}{|c|c|c|c|c|c|c|c|}
\hline $\begin{array}{c}\text { Мальтоза, } \\
\%\end{array}$ & Модель & $\begin{array}{c}\langle R\rangle, \\
\AA\end{array}$ & $m$ & $\begin{array}{c}d, \\
\AA\end{array}$ & $\begin{array}{c}D, \\
\AA\end{array}$ & $\begin{array}{c}\rho_{\mathrm{PH}}, \\
10^{10} \mathrm{~cm}^{-2}\end{array}$ & $\chi^{2}$ \\
\hline \multirow{2}{*}{20} & ПМ & $207 \pm 2$ & $18 \pm 1$ & $52,0 \pm 0,8$ & $19,7 \pm 0,3$ & $10,7 \pm 0,3$ & 0,65 \\
& РФФ & $208 \pm 3$ & $18 \pm 1$ & $51,3 \pm 0,8$ & $19,9 \pm 0,3$ & $10,7 \pm 0,3$ & 0,66 \\
\hline \multirow{2}{*}{25} & ПМ & $164 \pm 2$ & $19 \pm 1$ & $48,1 \pm 0,7$ & $20,7 \pm 0,2$ & $10,9 \pm 0,2$ & 0,37 \\
& РФФ & $165 \pm 2$ & $19 \pm 1$ & $47,8 \pm 0,7$ & $20,7 \pm 0,2$ & $10,9 \pm 0,3$ & 0,37 \\
\hline \multirow{2}{*}{30} & ПМ & $149 \pm 2$ & $19 \pm 1$ & $36,3 \pm 0,6$ & $22,7 \pm 0,2$ & $11,6 \pm 0,1$ & 0,42 \\
& РФФ & $148 \pm 2$ & $19 \pm 1$ & $36,6 \pm 0,6$ & $22,6 \pm 0,2$ & $11,6 \pm 0,2$ & 0,42 \\
\hline
\end{tabular}


Таблица 3. Расчет на основе полной модели (ПМ) и модели РФФ параметров везикулярной системы ФТНС с двухступенчатой функцией распределения электронов (см. рис. 1, б)

\begin{tabular}{|c|c|c|c|c|c|c|c|c|}
\hline $\begin{array}{c}\text { Мальтоза, } \\
\%\end{array}$ & Модель & $\begin{array}{c}R, \\
\AA\end{array}$ & $m$ & $\begin{array}{c}d, \\
\AA\end{array}$ & $\begin{array}{c}D, \\
\AA\end{array}$ & $\begin{array}{c}L, \\
\AA\end{array}$ & $\begin{array}{c}\rho_{\mathrm{PH},}, \\
10^{10} \mathrm{~cm}^{-2}\end{array}$ & $\chi^{2}$ \\
\hline \multirow{2}{*}{20} & ПМ & $206 \pm 3$ & $18 \pm 1$ & $44,4 \pm 0,4$ & $27,3 \pm 0,2$ & $10,5 \pm 0,9$ & $12,4 \pm 0,2$ & 0,70 \\
& РФФ & $205 \pm 3$ & $18 \pm 1$ & $44,9 \pm 0,4$ & $27,1 \pm 0,2$ & $11,2 \pm 0,9$ & $12,4 \pm 0,2$ & 0,70 \\
\hline \multirow{2}{*}{25} & ПМ & $161 \pm 2$ & $18 \pm 1$ & $40,8 \pm 0,3$ & $25,4 \pm 0,2$ & $2,9 \pm 0,3$ & $11,9 \pm 0,2$ & 0,36 \\
& РФФ & $160 \pm 2$ & $18 \pm 1$ & $40,0 \pm 0,3$ & $26,4 \pm 0,1$ & $3,2 \pm 0,2$ & $11,9 \pm 0,2$ & 0,36 \\
\hline \multirow{2}{*}{30} & ПМ & $147 \pm 2$ & $19 \pm 1$ & $37,0 \pm 0,3$ & $22,7 \pm 0,2$ & $1,0 \pm 0,1$ & $11,5 \pm 0,4$ & 0,42 \\
& РФФ & $146 \pm 2$ & $19 \pm 1$ & $37,0 \pm 0,2$ & $22,6 \pm 0,7$ & $1,0 \pm 0,1$ & $11,5 \pm 0,4$ & 0,41 \\
\hline
\end{tabular}
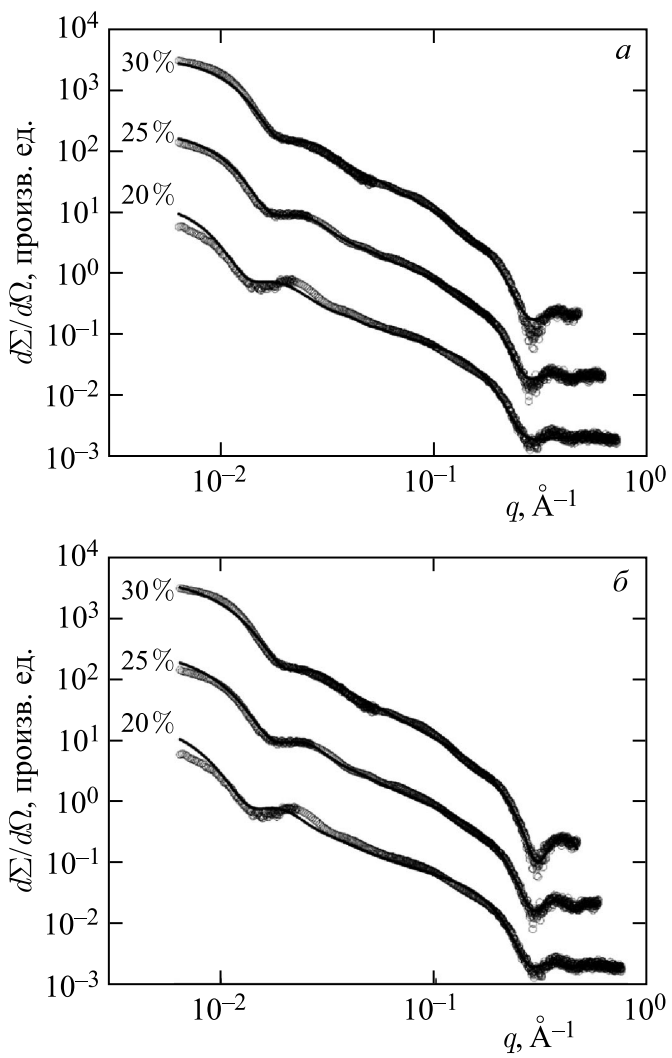

Рис. 2. Рассчитанные и экспериментально определенные спектры МУРР на образцах везикул ФТНС в водных растворах мальтозы с концентрацей 20, 25 и $30 \%$. Расчет сделан с использованием одноступенчатой $(a)$ и двухступенчатой (б) моделей бислоя 
Из табл. 2 и 3 видно, что результаты расчетов на основе полной модели (ПМ) и модели РФФ очень близки и отличаются не больше, чем на $1 \AA$ А. Это свидетельствует о применимости метода РФФ даже в случае достаточно малых размеров везикул и подтверждает возможность использования РФФ для исследования дальнейшей структуры ФТНС и лекарственных средств на этой основе.

Для обеих моделей бислоя наблюдается существенное уменьшение (до 1,4 раз) размера везикул ФТНС при увеличении концентрации мальтозы с 20 до $30 \%$. При этом полидисперсность среднего радиуса меняется незначительно и остается достаточно низкой: 22-23\%. Видно, что обе модели бислоя дают в целом неплохое согласие с экспериментальными данными и близкие значения $\chi^{2}$. Рассчитанные кривые, полученные в рамках двух моделей, практически не различаются в левой части спектра. Однако осцилляции в правой части спектра лучше воспроизводятся в рамках двухступенчатой модели. Поэтому результаты, полученные на этой основе, представляются более реалистичными. Толщина бислоя $d$ уменьшается от 44 до $37 \AA$ А с ростом концентрации мальтозы от 20 до $30 \%$. Область полярных голов $h=(d-D) / 2$ также уменьшается приблизительно на $20 \%$ : от 8,6 до 7,5 А․ Сильное уменьшение области $L$ углеводородных цепочек $\mathrm{CH}_{3}$ (с $11 \AA$ при концентрации мальтозы $20 \%$ до 1 А̊ при концентрации $30 \%$ ) можно объяснить перекрытием областей $\mathrm{CH}_{3}$ двух липидных слоев в центре бислоя. Тем самым с ростом концентрации мальтозы снижается вклад области $\mathrm{CH}_{3}$ в расчетную интенсивность МУРР. Соответственно, размеры бислоя $d$ и области $D$ углеводородных цепочек для случая 30\%-й концентрации мальтозы, полученные в рамках двухступенчатой модели, практически совпадают со значениями, полученными на основе одноступенчатой модели, не учитывающей вклад области $\mathrm{CH}_{3}$.

\section{ВЫВОДЫ}

Проведенные на установке ДИКСИ эксперименты методом МУРР при концентрации фосфолипидной транспортной наносистемы в воде от 25 до $37,5 \%$ подтвердили, что терапевтические концентрации раствора лиофилизата ФТНС в воде (25\%) позволяют измерять спектры малоуглового рассеяния от образцов без существенных искажений и с достаточным контрастом, что обеспечивает возможность проводить на этой основе анализ структуры везикулярной системы как ФТНС, так и лекарственных веществ на основе ФТНС.

Основным результатом анализа экспериментальных данных МУРР, полученных на установке ДИКСИ, является определение базовых структурных параметров везикулярной системы ФТНС в зависимости от концентрации мальтозы в водном растворе. Показано, что средний размер везикул меняется 
от $410 \AA$ при концентрации мальтозы $20 \%$ до $280 \AA$ при концентрации $30 \%$. Показано, что система везикул ФТНС имеет низкую полидисперсность (22$23 \%)$ при всех исследованных концентрациях мальтозы. Определены параметры липидного бислоя, что позволяет рассчитывать гидрофобный объем везикулярного переносчика лекарственного вещества, доступный для размещения водонерастворимых лекарств. Гидрофобный объем одной везикулы имеет максимальное значение $14,55 \cdot 10^{6} \AA^{3}$ при концентрации ФТНС в воде $25 \%$. Увеличение концентрации ФТНС в воде до $37,5 \%$ приводит к уменьшению гидрофобного объема до $6,16 \cdot 10^{6} \AA^{3}$.

Полученные результаты будут использованы для исследования структуры нанолекарств на основе ФТНС.

Исследование выполнено при финансировании грантом РНФ 14-12-00516.

\section{ЛИТЕРАТУРА}

1. Das S., Chaudhury A. // AAPS PharmSci. Tech. 2011. V.12. P. 62.

2. Martins S. et al. // Intern. J. Nanomedicine. 2007. V.2. P. 595.

3. Стрекалова О. С. Автореф. дис.... канд. биол. наук. М., 2010.

4. Арчаков А. И. и др. Пат. № 2463057, РФ, МПК А61К 31/685 А61К 9/127 А61К $1 / 16$ B82B 1/00.

5. Ipatova O. M. et al. // Biomed. Chemistry. 2010. V.4. P. 82.

6. Kiselev M. A. et al. // J. Pharmaceut. and Biomed. Analysis. 2015. V. 114. P. 288.

7. Zhabitskaya E. et al. // EPJ Web Conf. 2016. V. 108. P. 02047.

8. Zemlyanaya E. V. et al. // J. Phys.: Conf. Ser. 2016. V.724. P. 012056.

9. Киселев М.А. // ЭЧАЯ. 2011. Т. 42. С. 578.

10. Свергун Д. И., Фейгин Л.А. Рентгеновское и нейтронное малоугловое рассеяние. М.: Наука, 1986. 276 c.

11. Kucerka N., Mu-Ping Nieh, Katsaras J. Small-Angle Scattering from Homogenous and Heterogeneous Lipid Bilayers // Advances in Planar Lipid Bilayers and Liposomes. Burlington: Elsevier, Acad. Press, 2010. V. 12. P. 201.

12. Kiselev M. A., Lombardo D. // Biochimica et Biophysica Acta. 2017. V. 1861. P. 3700.

13. Kiselev M. A. et al. // Eur. Biophys. J. 2006. V.35. P. 477.

14. Kiselev M. A. et al. // Nucl. Instr. Meth. A. 2001. V.470. P. 409.

15. Kiselev M. A. et al. // J. Alloys Compd. 2001. V.328. P. 71.

16. Kiselev M. A. et al. // Chem. Phys. Lipids. 2003. V. 123. P. 31.

17. Kiselev M. A. et al. // Colloids Surf., A: Physicochem. Eng. Aspects. 2005. V. 256. P. 1.

18. Киселев М.А. и др. // Поверхность. Рентгеновские, синхротронные и нейтронные исслед. 2003. № 11. С. 20.

19. Zemlyanaya E. V. et al. // Crystallography Rep. 2006. V.51, No. 1. P. S22. 
20. Земляная E. В. и др. // Поверхность. Рентгеновские, синхротронные и нейтронные исслед. 2008. № 11. С. 14.

21. Kiselev M. A. et al. // Appl. Phys. A. 2014. V. 116. P. 319.

22. Киселев М. А. и др. // Кристаллография. 2015. Т. 60, № 1. С. 140.

23. Ostanevich Y.M. // Makromol. Chem., Macromol. Symp. 1988. V. 15. P. 91.

24. Dymov S. N. et al. // Nucl. Instr. Meth. A. 2000. V.440. P. 431.

25. Zhabitskaya E. I., Zhabitsky M. V. // Lect. Notes Comput. Sci. Springer, 2012. V. 7125. P. 328 .

26. Жабиикая Е. И., Жабицкий М. В. // Матем. моделирование. 2012. Т. 24, № 12. С. 33.

Получено 31 мая 2017 г. 
Редактор Е. В. Григорьева

Подписано в печать 05.07.2017.

Формат $60 \times 90 / 16$. Бумага офсетная. Печать офсетная.

Усл. печ. л. 0,81. Уч.-изд. л. 0,99. Тираж 210 экз. Заказ № 59158.

Издательский отдел Объединенного института ядерных исследований 141980 , г. Дубна, Московская обл., ул. Жолио-Кюри, 6.

E-mail: publish@jinr.ru

www.jinr.ru/publish/ 